Rapid Reviews COVID-19

\title{
Review 2: "Inhibition of
}

SARS-CoV-2 infection in

\section{human cardiomyocytes by}

targeting the Sigma-1

receptor disrupts

cytoskeleton architecture

and contractility"

Kenji Hashimoto ${ }^{1}$

${ }^{1}$ Chiba University: Chiba Daigaku, Clinical Neuroscience, Japan

Published on: Mar 29, 2021

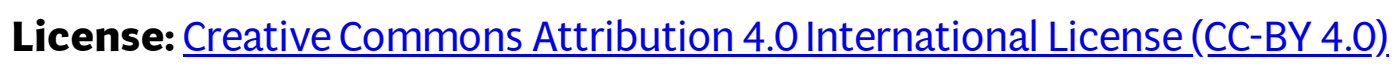




\section{$\underline{\text { RR:C19 Evidence Scale rating by reviewer: }}$}

- Reliable. The main study claims are generally justified by its methods and data. The results and conclusions are likely to be similar to the hypothetical ideal study. There are some minor caveats or limitations, but they would/do not change the major claims of the study. The study provides sufficient strength of evidence on its own that its main claims should be considered actionable, with some room for future revision.

$* * * * * * * * * * * * * * * * * * * * * * * * * * * * * * * * * * * * * * *$

\section{Review:}

Using hiPSC-CM (human cardiomyocytes derived from iPSCs), the authors examined the effects of NE-100, a selective sigma-1 receptor antagonist, on SARS-CoV-2 infection and viral replication. NE-100 reduced cytokine release and cell death associated with replication. In contrast, NE-100 compromised cytoskeleton integrity and reduced bearing frequency, causing contractile impairment. The experiments using hiPSC-CM were conducted very well. These data suggest that NE-100 could be a useful drug for SARS-CoV-2 infection although further in vivo study of NE-100 is needed. Claims are generally supported by the data and methods used. However, the following concerns should be addressed before publication.

\section{Major concern:}

- The authors cited the article on fluvoxamine in patients with SARS-CoV-2 replication (Lenze et al, JAMA 2020). Fluvoxamine is known to be a sigma receptor agonist. In this study, the authors selected NE-100 as a selective sigma-1 receptor antagonist. At present, it is unclear if a sigma-1 receptor antagonist or agonist could be better for inhibiting SARS-CoV-2 replication (discussed by Hashimoto K. Eur. Arch. Psychiatry Clin. Neurosci. 2021). Additional experiments using sigma-1 receptor agonists (i.e., SA-4503, fluvoxamine) in the same assay should be needed.

\section{Minor concerns}

- Previous articles (i.e., Vela JM. Front. Pharmacol. 2020; Hashimoto K. Eur. Arch. Psychiatry Clin. Neurosci. 2021) on the role of the sigma-1 receptor for COVID-19 
should be cited in the introduction.

- A multicenter observational study showed that haloperidol (potent sigma-1 receptor antagonist) use may not be associated with the risk of intubation or death (Hoerten $\mathrm{N}$, et al. PLOS One 2021). This paper suggests that a sigma-1 receptor antagonist may not be useful for COVID-19. Please discuss this paper.

- A new paper showed beneficial effects of fluvoxamine (a sigma-1 receptor agonist) for early treatment of COVID-19 (Seftel D, et al. Open Forum Infect Dis 2021). Please discuss this paper. 